\title{
Semiconductor sensors for dosimetry of epithermal neutrons
}

\author{
P. G. Litovchenko' ${ }^{1}$, R. Moss ${ }^{2}$, F. Stecher-Rasmussen ${ }^{2}$, K. Appelman², L. I. Barabash ${ }^{1}$, \\ T. I. Kibkalo', V. F. Lastovetsky ${ }^{1}$, A. P. Litovchenko ${ }^{1}$, M. B. Pinkovska ${ }^{1}$. \\ ${ }^{I}$ SC Institute for Nuclear Research of NASU, 47 prospect Nauki, 252028 Kiev, Ukraine. \\ ${ }^{2}$ ECN, Westerduinweg 3, P. O. Box 1,1755 ZG Petten, The Netherlands.
}

\begin{abstract}
Minimum energy of neutron to displace atoms in silicon crystals are equal to $200 \mathrm{eV}$. Due to this fact testing our $p-i-n$ diodes under irradiation by the epithermal neutrons was carried out. The more advanced $p-i-n$ diodes on the base of high purity silicon were used at present work, and, as a result, we have obtained considerably more sensitive sensors for more wide range of neutron doses. The sensitivity of sensors is $0.14 \mathrm{~V} / \mathrm{Gy}$ for average neutron energy of $24 \mathrm{keV}$.
\end{abstract}

Keywords: dosimetry, semiconductor detectors, epithermal neutrons, silicon, converter.

Paper received 17.05.98; revised manuscript received 26.06.98; accepted for publication 12.07.98.

\section{Introduction}

The measurement of epithermal neutron flux is very significant task for Boron Neutron Capture Therapy (BNCT). In this paper semiconductor sensors were used for epithermal neutron flux measurement. These semiconductor sensors were produced by us. These are $p-i-n$ diodes and semiconductor detectors with ${ }^{235} \mathrm{U}$ converter.

Possibilities for application of fast neutron dosimeters using the $p-i-n$ diodes as a base have been shown earlier [1]. The principle of detection is based on the creation of radiation defects in semiconductors under the influence of fast neutrons, which change the electrical parameters (a diode voltage drop increases for a fixed current). It is known that the minimum energy of neutron to displace atoms in silicon crystals is equal to $\sim 200 \mathrm{eV}$. Due to this fact there were all reasons to test the $p-i-n$ diodes prepared by us under irradiation in the epithermal neutron energy range.

\section{Experimental results}

At the beginning of investigation, our colleagues and we have made the detail analysis of all available dates, such as the KERMA damage and the total KERMA for silicon [2]. The results of experimental verification for monoenergetic neutrons in the energy range from 90 to $900 \mathrm{keV}$ were obtained in [2]. In spite of the complicated view of the KERMA damage dependence, as evident from these data, a proper coincidence between the experimental results and theoretical calculations of KERMA by Monte Karlo method was obtained. The presence of some hollow in the neutron energy range from 100 to $300 \mathrm{eV}$ partly makes difficulties to use the $p-i-n$ diodes as tissue-equivalent sensors in practically used energy range of epithermal neutrons. There is doubtless interest to test the offered by us semiconductor sensors intended for measurements of total tissue dose from the extremely complicated spectrum of Petten High Flux Reactor, which was used in BNCT [3]. From these data, the fast neutrons $(>10 \mathrm{keV})$ make a considerable and undesirable contribution to damage KERMA. In our work [4], it was shown that $p-i-n$ diodes, which were calibrated with a known energy, could be used to determine tissue doses, received in the courses of irradiation by neutrons with an unknown energy. Proceeding from this fact, we were able to determine a sensitivity of our sensors under the energy of $24 \mathrm{keV}$ (the channel HB12) and intensity of $1.23 \cdot 10^{12} \mathrm{n} / \mathrm{m}^{2} \cdot \mathrm{sec}$.

The changes of a direct voltage drop $(\Delta V)$ for $p-i-n$ diodes under fixed current of $1 \mathrm{~mA}$ (at irradiation time $85 \mathrm{~min}$, 


\section{P.G. Litovchenko et al.: Semiconductor sensors for dosimetry of...}

$E_{n}=24 \mathrm{keV}$, tissue KERMA $1 \mathrm{~Gy}=5 \cdot 10^{15} \mathrm{n} / \mathrm{m}^{2}$ ) were measured. The average changes of the direct voltage drop is $\Delta V=0.175 \mathrm{~V}$ and the received dose is $\Phi=1.25 \mathrm{~Gy}$. The average sensitivity of sensors was $\Delta V / \Phi=0.140 \mathrm{~V} / \mathrm{Gy}$.

Dose dependences of $\Delta V$ were obtained by means of additional irradiation of the same diodes up to the total 16 hours. Dose dependencies are presented in Fig. 1 for the sensors of numbers $2 \mathrm{n}$ and $3 \mathrm{n}$ with the best and the worst sensitivities, respectively. As one can see from Fig. 1, the changes of a direct voltage drop are very good fits to linear dependence $\Delta V v s \Phi$ in the range of used irradiation doses.

Mentioned sensors were calibrated in the channel HB12, and then irradiated in the channel HB11 for $1.8 \cdot 10^{3} \mathrm{sec}$. Our sensors give the average tissue dose of neutrons as much as $\Phi=(2.76 \pm 0.16)$ Gy per hour.

In this channel, besides neutrons, the gamma irradiation is presents, according to the HB11 channel technical characteristics, and gives the contribution to dose 1.2 Gy per hour. As the coefficient of discrimination for our neutron sensors in relation to the gamma irradiation is $\sim 10^{3}$ [2], then the determined above tissue dose entirely relates to the neutron spectrum of the channel HB 11. Proceeding from the HB11 technical characteristics one may appreciate the share of fast and epithermal neutrons into tissue KERMA. The epithermal neutron flux $(0,414 \mathrm{eV}-10 \mathrm{keV})$ is $3 \cdot 3 \cdot 10^{12} \mathrm{n} / \mathrm{m}^{2} \mathrm{sec}$. If we suppose that all neutrons in this spectrum possess the energy as much as $10 \mathrm{keV}\left(\mathrm{KERMA}=10^{16} \mathrm{n} / \mathrm{m}^{2}\right)$, then the dose will correspond to $\Phi=1.2$ Gy per hour. Under assumption that both the average energy and flux of fast neutrons are $0.5 \mathrm{MeV}\left(\mathrm{KERMA}=5.6 \cdot 10^{8}\right)$, and $4.7 \cdot 10^{7} \mathrm{n} / \mathrm{cm}^{2} / s(>10 \mathrm{keV})$ respectively, we obtained $\Phi=1.6 \mathrm{~Gy}$. These appraisals of ours coincide with obtained by us results on the total dose $(1.6+1.2)=2.8$ Gy and confirm an undesirable contribution of fast neutrons $(60 \%)$ in the total theraputical dose.

The epithermal neutron flux at the HB11 channel was measured also by the silicon barrier-surface detectors with

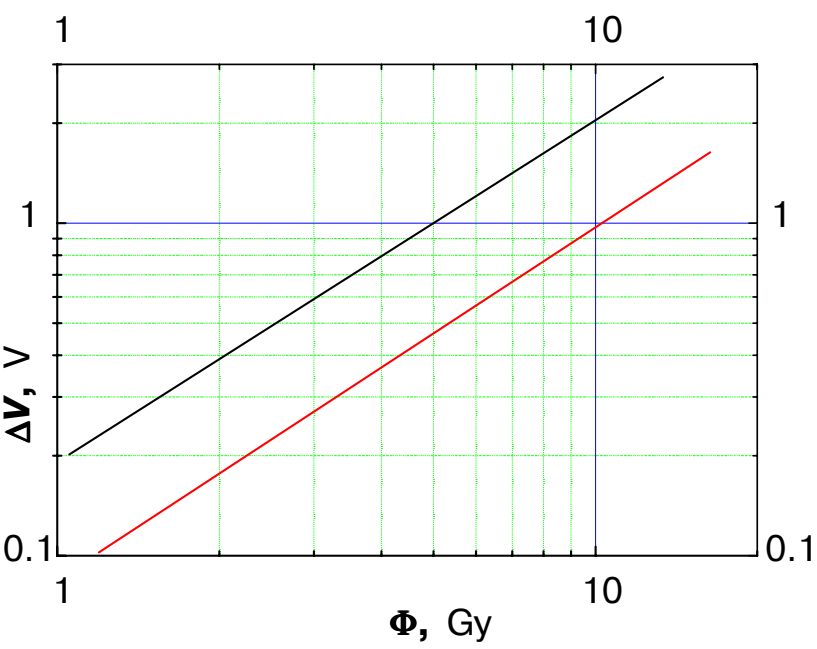

Fig. 1. Dose dependence as a function of change of voltage $(\Delta \mathrm{V})$ at $p$ - $i$ - $n$ diodes with big $(2 n)$ and little (3n) sensitivity under irradiation of neutron with energy of $24 \mathrm{keV}$. an attached ${ }^{235} \mathrm{U}$ converter. The converter as ${ }^{235} \mathrm{U}$ oxideprotoxide being enriched up to $99.92 \%$ with the thickness of $1.5 \cdot 10^{-4} \mathrm{~m}$, mass $10^{-6} \mathrm{~kg}$ and alpha activity of $81 \mathrm{~Bq}$ was fabricated on Al- sublayer. We established that the detectors efficiency to epithermal neutrons the (HB11 neutron spectrum) is $1.82 \cdot 10^{-5} \mathrm{imp} / \mathrm{n}$.

\section{Conclusions}

The use of $p-i-n$ diodes made on the base of high purity silicon for registration of epithermal neutrons with the coefficient of gamma ray discrimination $10^{3}$ is demonstrated. A good agreement of experimental data with theoretical calculations of the damage KERMA for silicon has been shown.

It is shown that $p-i-n$ diodes can serve as tissue-equivalent dosimeters in the neutron energy range from $300 \mathrm{eV}$ till $30 \mathrm{keV}$. The possibility of neutron flux monitoring by means of silicon detectors with ${ }^{235} \mathrm{U}$ converter was shown.

\section{References}

1. A. B. Rosenfeld, L. I.Barabash, P. G. Litovchenko, V. I. Khivrich, $P$-i-n Diode with a Wide Measurement Range of Fast Neutron Doses., Rad. Prot. Dos., 33(1/4), pp. 175-178 (1990).

2. M. G. Carolan, A. B. Rosenfeld, S. Wallace, H. Mariaty, G. J. Storr, V. I. Khivrich, R. Moss, B. J. Alien, Silicon Dosimeter Diode for BNCT Using Epithermal Neutron Sources. Proc. of 1-st Intern. Workshop on Accelerator-Based Neutron Sources for BNCT, I, September 11-14, 1994, Jacson, Wyoming, pp. 299-309.

3. Boron Neutron Capture Therapy, Proc. of Intern. Workshop and plenary meeting, Petten The Netherlands, 1991, Edited by D. Gabel and R. Moss, p.47.

4. V. I. Khivrich, M. D. Varentsov, P. G. Litovchenko, A. I. Anokhin, A. B. Rosenfeld, M. G. Carolan, O. S. Zinets, M. I. Reinhard, D. Alexiev, High Purity Silicon as a Basic Material for Manufacturing of Radiation Defects and Integral Neutron Dosimeters, IEEE Trans. on Nucl. Sci., NS-43, N6, pp. 2687-2692 (1996). 